\title{
Divergent Synthesis of Hydrophilic Dendrons Based on Tri(ethylene glycol) Spacers
}

\author{
Jie Song and Byoung-Ki Cho* \\ Department of Chemistry and Institute of Nanosensor and Biotechnology, Dankook University, Gyeonggi-Do 448-701, Korea \\ *E-mail:chobk@dankook.ac.kr \\ Received March 8, 2010, Accepted May 18, 2010
}

Key Words: Hydrophilic dendrons, Divergent synthesis, Tri(ethylene glycol) spacers, Phloroglucinol junctures

Dendritic molecules have received a great deal of attention because of their peculiar 3-D architecture, capable of confining and releasing certain drugs under certain conditions. ${ }^{1}$ As a prerequisite for biological applications, dendritic molecules must gain water solubility. To date researchers have achieved appropriate water solubility by using either charged ${ }^{2}$ or nonionic $^{3,4}$ moieties. Charged compounds, however, can cause cytotoxicity such as undesired cell lysis. Therefore, researchers have considered using neutral polymeric or oligomeric coils on dendritic compounds as an alternative for in vivo applications. Particularly, oligo- or poly(ethylene glycol) (PEG) could be a useful candidate for endowing these compounds with water solubility. ${ }^{4}$

Herein, we report on the synthesis of hydrophilic polyether dendrons from first to third generation. As Scheme 1 shows, the dendrons possess tri(ethylene glycol) (TEG) spacers and phloroglucinol branching junctures. Because of their oxygenrich compositions and multiple hydroxyl termini, the dendrons are hydrophilic enough to be water-soluble. Indeed, the third generation favors water over ethyl acetate in the extraction. To synthesize dendrons, we adopted a divergent route consisting of i) the tosylation of terminal alcohols and ii) the Williamson etherification as the activation and coupling steps, respectively. In the synthesis, the key molecule is the $\mathrm{AB}_{2}$-type monomeric unit (5) where A and B are phenol and aliphatic alcohol, res-

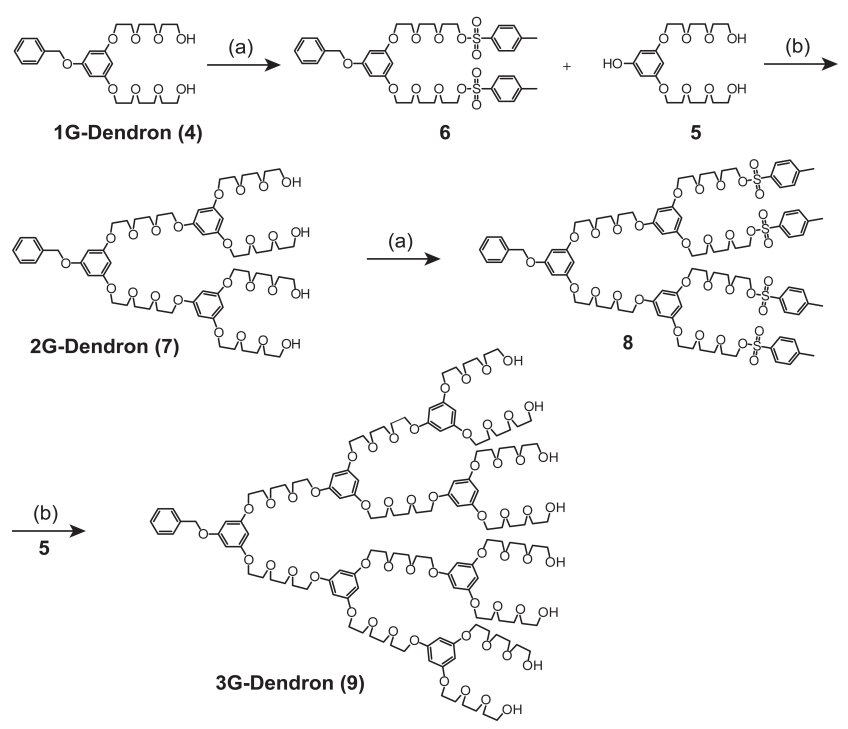

Scheme 1. Divergent synthesis of the hydrophilic dendrons (nG-Dendron, $\mathrm{n}=1,2$, and 3). Reagents: (a) TsCl, pyridine, and $\mathrm{CH}_{2} \mathrm{Cl}_{2}$; (b) $\mathrm{K}_{2} \mathrm{CO}_{3}$, KI, and $\mathrm{CH}_{3} \mathrm{CN}$. pectively. In particular, these alcohols are well-known to have different $\mathrm{p} K_{\mathrm{a}}$ values. In the presence of potassium carbonate $\left(\mathrm{K}_{2} \mathrm{CO}_{3}\right)$, the phenol group is completely deprotonated, but the aliphatic alcohol remains intact. As a result of this selective deprotonation, only the phenol group reacts during the coupling step. On the other hand, the two unreacted aliphatic alcohols can be converted easily into tosylate as a good leaving group in the next step. This monomer design eliminates an involvement of a tedious benzyl protection/deprotection (commonly used in dendrimer synthesis) ${ }^{5}$ sequence during the generation growth.

The synthesis began with the preparation of monobenzylated phloroglucinol. Although the monobenzylation seems to be done simply by one-step etherification under potassium carbonate, the compound actually was prepared by a three-step reaction: i) acetylation, ii) benzylation, and iii) hydrolysis (Scheme S1). This is because one-step synthesis from phloroglucinol produced an undesirable $C$-benzylated product ${ }^{6}$ and gave only a $9 \%$ yield. $^{7}$ Thus, to avoid this byproduct, the electron-density of phloroglucinol should be reduced by acetylation. ${ }^{8}$ In the next benzylation step, to obtain the monobenzylated product, 1 molar equiv. of water was added dropwise into a mixture of sodium hydride and benzyl bromide. ${ }^{9}$ In this reaction, the phenoxides formed by the hydrolysis of the acetoxy groups react with benzyl bromide. The remaining acetyl groups were removed under a $5 \mathrm{M} \mathrm{KOH}$ solution, resulting in 5-benzyloxy-resorcinol (3). The overall yield was approximately $43 \%$, which is much bigger than that ( $9 \%$ ) from the one-step synthesis. In the ${ }^{1} \mathrm{H}-\mathrm{NMR}$ spectrum, the two distinct aromatic protons of the phloroglucinol ring were observed at $5.97 \mathrm{ppm}$ and $6.08 \mathrm{ppm}$, respectively, and any proton resonance for the $C$-benzylated product was not exhibited. The first generation dendron (1G-Dendron) was synthesized by the Williamson etherification of compound $\mathbf{3}$ with monotosylated TEG with a yield of $87 \%$. The next debenzylation, using $10 \% \mathrm{Pd} / \mathrm{C}$ and $\mathrm{H}_{2}$, led to the monomeric unit (5).

The generations up to the third were grown successfully by a divergent method (Scheme 1). As noted previously, this divergent route consists of tosylation and the Williamson etherification. These elemental reactions are well-known to be conventional and free of side reactions. Most tosylation and etherification reactions showed high reaction yields of more than $70 \%$. However, the third generation (3G-Dendron) gave only a $27 \%$ yield, perhaps because of the steric effect of the multibranches. The obtained dendrons (nG-Dendron) were characterized by ${ }^{1} \mathrm{H}$ - and ${ }^{13} \mathrm{C}$-NMR, gel permeation chromatography (GPC), elemental analysis, and matrix-assisted laser desorption/ ionization time-of-flight mass spectrometry (MALDI-TOF MS). 
Figure 1(a-c) represents the ${ }^{1} \mathrm{H}-\mathrm{NMR}$ spectra of the dendrons. As the generation increases, the intensities of the TEG protons exceed those of the aromatic protons. Also, the integral ratios of the two ortho-protons (assigned as $c$ ) to the benzyloxy group versus the other phloroglucinol protons $(d)$ are approximately $0.5,3.5$, and 9.5 for the first, second, and third generations, respectively, consistent with the theoretical values. As well as

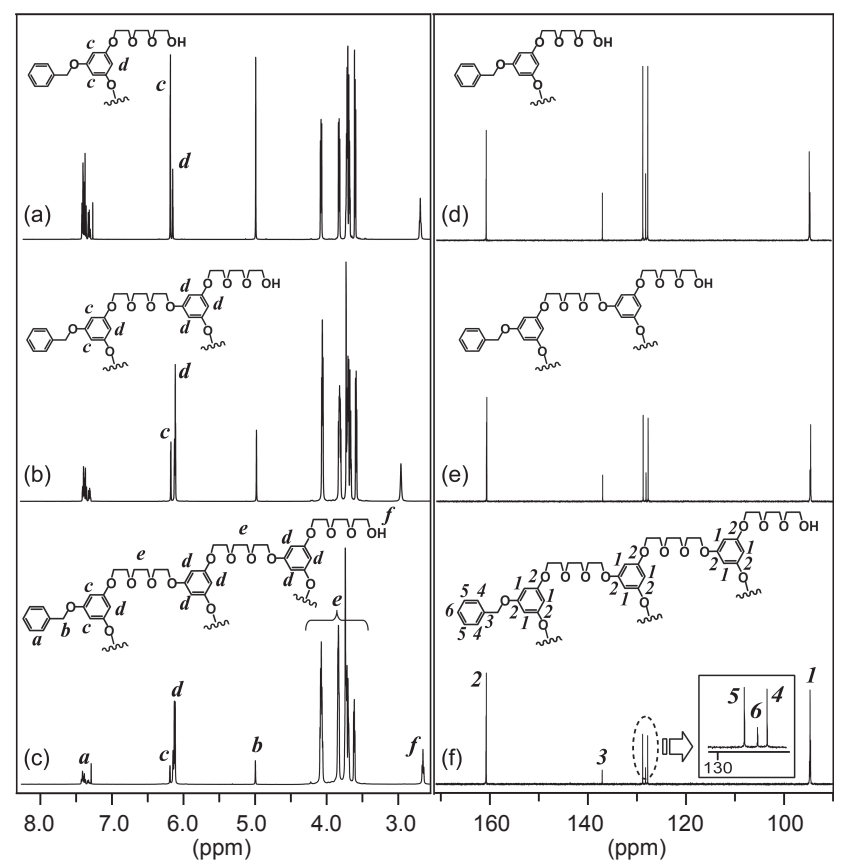

Figure 1. Proton (left) and carbon (right) NMR spectra of (a,d) 1GDendron, (b,e) 2G-Dendron, and (c,f) 3G-Dendron.
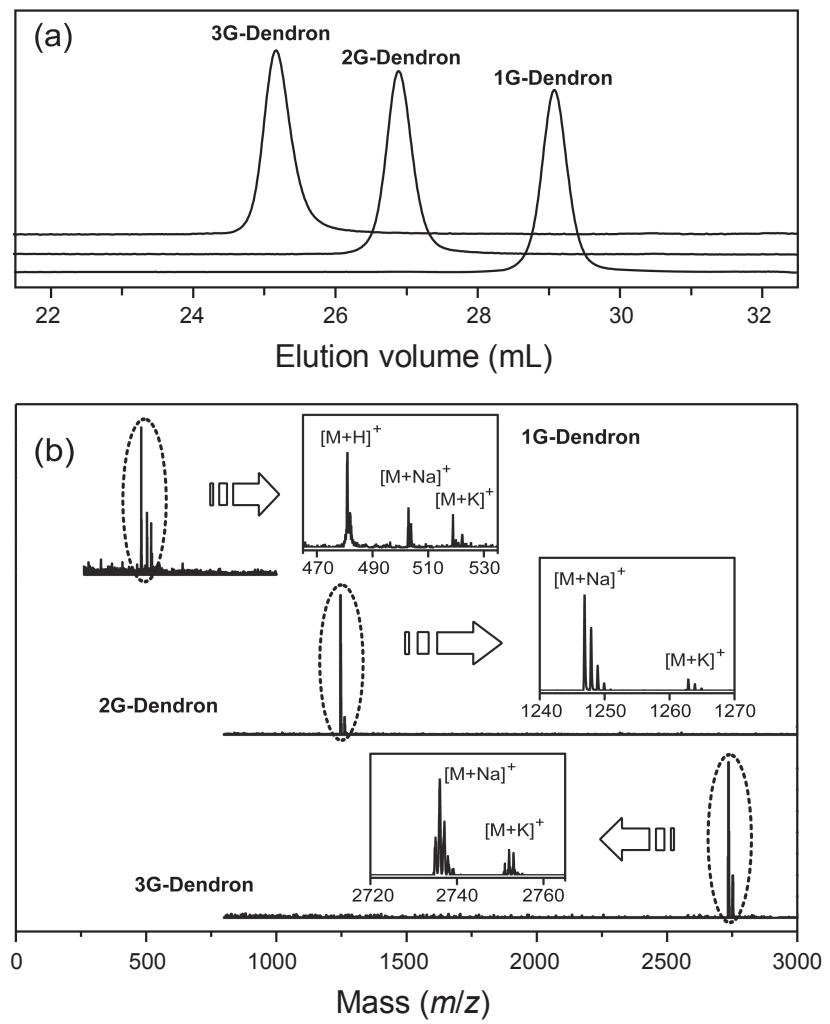

Figure 2. (a) GPC elugrams, and (b) MALDI-TOF MS spectra. the ${ }^{1} \mathrm{H}-\mathrm{NMR}$ results, six distinct aromatic carbon resonances were observed in all the ${ }^{13} \mathrm{C}$-NMR spectra, which corroborate the successful synthesis of the designed molecular structures (Figure 1(d-f)).

An analysis of the GPC and MALDI-TOF MS data revealed no structural defect, which can occur from incomplete coupling in divergent methods. ${ }^{10}$ In the GPC data, the polydispersity values $\left(\mathrm{M}_{\mathrm{w}} / \mathrm{M}_{\mathrm{n}}\right)$ for the second and third generations were less than 1.03, suggesting high purity (Figure 2(a)). In Figure 2(b), the MALDI-TOF MS spectra provide direct evidence of no structural defect. The mass spectrum of 1G-Dendron shows three signals corresponding to the $\mathrm{H}, \mathrm{Na}$, and $\mathrm{K}$ adducts of the molecular ions; and 2G-Dendron and 3G-Dendron exhibit Na and $\mathrm{K}$ adducts. And the measured molecular masses of $\mathbf{1 G -}$ Dendron, 2G-Dendron and 3G-Dendron are $479.9 \mathrm{~g} / \mathrm{mol}$, $1223.9 \mathrm{~g} / \mathrm{mol}$, and $2713.1 \mathrm{~g} / \mathrm{mol}$, respectively, which closely match their theoretical molecular masses. ${ }^{11}$

In summary, we prepared a series of hydrophilic dendrons based on TEG spacers and phloroglucinol branching junctures. A divergent synthetic route consisting of tosylation and the Williamson etherification as the activation and coupling steps, respectively, was applied. Iterating the two-step reactions enabled the generation to reach the third one. Structural analysis demonstrated that the obtained dendrons showed their designed molecular structure with no structural defects.

Acknowledgments. This research was supported by the Graduate Research Assistantship of Dankook University.

Supporting Information. The supporting Information is available on request from the corresponding author (E-mail: chobk@dankook.ac.kr).

\section{References and Notes}

1. (a) Jansen, J. F. G. A.; de Brabander-van den Berg, E. M. M.; Meijer, E. W. Science 1994, 266, 1226. (b) Fuchs, S.; Otto, H.; Jehle, S.; Henklein, P.; Schlüter, A. D. Chem. Commun. 2005, 1830.

2. (a) Hawker, C. J.; Wooley, K. L.; Fréchet, J. M. J. J. Chem. Soc., Perkin Trans. 1 1993, 1287. (b) Cheng, C.; Schmidt, M.; Zhang, A.; Schlüter, A. D. Macromolecules 2007, 40, 220.

3. Ashton, P. R.; Boyd, S. E.; Brown, C. L.; Jayaraman, N.; Stoddart, J. F. Angew. Chem. Int. Ed. 1997, 36, 732.

4. (a) Tian, L.; Hammond, P. T. Chem. Mater. 2006, 18, 3976. (b) Feng, X.; Taton, D.; Borsali, R.; Chaikof, E. L.; Gnanou, Y. J. Am. Chem. Soc. 2006, 128, 11551. (c) Gillies, E. R.; Fréchet, J. M. J. J. Am. Chem. Soc. 2002, 124, 14137. (d) Huang, Z.; Liu, L.; Lee, E.; Lee, M. Bull. Korean Chem. Soc. 2008, 29, 1485. (e) Kim. H.-Y.; Lee, B.-I.; Cho, B.-K. Macromol. Rapid Commun. 2008, 29, 1758. (f) Kim. H.-Y.; Song, J.; Kim, S.-H.; Lee, E.; Lee, J.-K.; Zin, W.-C.; Cho, B.-K. Chem. Eur. J. 2009, 15, 8683.

5. (a) Grayson, S. M.; Fréchet, J. M. J. Chem. Rev. 2001, 101, 3819. (b) Chow, H.-F.; Chan, I. Y.-K.; Mak, C. C.; Ng, M.-K. Tetrahedron 1996, 52, 4277. (c) Nithyanandhan, J.; Jayaraman, N. J. Org. Chem. 2002, 67, 6282.

6. Deme, E. J. Org. Chem. 1976, 41, 3769.

7. Curtis. W. D.; Stoddart, J. F. J. Chem. Soc., Perk. Trans. I 1977, 785.

8. Kawamoto, H.; Nakatsubo, F.; Murakami, K. Synth. Commun. 1981, 11,853.

9. Nagvekar, D. S.; Gibson, H. W. Org. Prep. Proced. Int. 1997, 29 , 240.

10. Chow, H.-F.; Wang, Z.-Y.; Lau, Y.-F. Tetrahedron 1998, 54, 13813.

11. The theoretical molecular masses for 1G-Dendron, 2G-Dendron and 3G-Dendron are $480.2 \mathrm{~g} / \mathrm{mol}, 1224.6 \mathrm{~g} / \mathrm{mol}, 2713.3 \mathrm{~g} / \mathrm{mol}$, respectively. The measured molecular masses were obtained from the highest peaks in the spectra. 